\title{
Quark mass dependence of pseudo-scalar masses and coupling constants
}

\author{
$\mathrm{qq}+\mathrm{q}$ Collaboration \\ F. Farchioni ${ }^{\mathrm{a}}$, C. Gebert ${ }^{\mathrm{b}}$, I. Montvay ${ }^{\mathrm{b}}$, E. Scholz ${ }^{\mathrm{b}}$, L. Scorzato $^{\mathrm{b}}$. \\ anstitut für Theoretische Physik, Universität Münster, Wilhelm-Klemm-Str. 9, \\ D-48149 Münster, Germany \\ b Deutsches Elektronen-Synchrotron DESY, Notkestr. 85, D-22603 Hamburg, Germany
}

The dependence of pseudo-scalar masses and decay constants on the sea and valence quark masses is investigated in the pseudo-Goldstone boson sector of QCD with two light quark flavours. The sea quark masses are at present in the range $\frac{1}{3} m_{s} \leq m_{u d} \leq \frac{2}{3} m_{s}$ whereas the valence quark masses satisfy $\frac{1}{2} m_{\text {sea }} \leq m_{\text {val }} \leq 2 m_{\text {sea }}$. The values of the Gasser-Leutwyler low energy constants $L_{4}, L_{5}, L_{6}$ and $L_{8}$ are estimated. The computation is done with the Wilson-quark lattice action at gauge coupling $\beta=5.1$ on $16^{4}$ lattices. $\mathcal{O}(a)$ effects are taken into account by applying chiral perturbation theory for the Wilson lattice action as proposed by Rupak and Shoresh.

\section{Introduction}

Monte Carlo simulations of QCD with dynamical quarks are done in most cases at relatively large quark masses (typically two quark flavours with $\left.m_{u d}>m_{s} / 2\right)$. This makes the extrapolation to the physical point $m_{u d} \simeq m_{s} / 20$ rather uncertain. The extrapolation is done by using (PQ)ChPT - typically to NLO (1-loop) order. Estimates [12] show that one should perform simulations in the range $m_{u d}<m_{s} / 4, m_{s} / 3$ in order to see the expected logarithmic dependence. Matching the predicted functional dependence is a crucial test for Lattice QCD.

Until recently the comparison between lattice data and ChPT was not satisfactory. In a recent paper 3 we suggested that the agreement can be found when going to light enough dynamical quarks. Here we supplement some integration of the analysis of [3] and provide some further comments.

\section{Simulations}

For our simulation we used the algorithm described in [4] and further improved as in 3 and references therein. In our range of parameters we found that the cost for producing one indepen- dent gauge field configuration roughly goes as:

Cost $\simeq F\left(a m_{q}\right)^{-2} \Omega$,

where $a m_{q}$ is the quark mass in lattice unit, $\Omega$ is the number of lattice points and the factor $F \simeq 10^{7}$ flop for the plaquette, but one order of magnitude smaller for $m_{\pi}$ and $f_{\pi}$.

We produced three sets of $O(1000)$ thermalized configurations for $N_{f}=2$ unimproved Wilson fermions at $\mathrm{Vol}=16^{4}, \beta=5.1$ and $\kappa=$ $0.176,0.1765,0.177$. From these points we extrapolated a value of $r_{0} / a$ at $\kappa_{c r}=0.1773(1)$ which is $r_{0}\left(\kappa_{c r}\right) / a=2.65(7)$. This corresponds to an UV cutoff $a=0.189(5) \mathrm{fm} \simeq(1.04 \mathrm{GeV})^{-1}$, and to a physical volume $L \simeq 3 \mathrm{fm}$. In those three points we found a ratio $m_{q} / m_{s}$ equal to $0.68,0.59$ and 0.35 respectively. Here $m_{q}$ represents the sea quark mass defined as $M_{r}=\left(m_{\pi} r_{0}\right)^{2}$, when the strange quark mass corresponds to $M_{r}=3.1$. For the lightest valence quarks the ratios $m_{q}^{v a l} / m_{s}$ become respectively $0.37,0.34$ and 0.17 . Even with these small quark masses finite volume effects are expected to be under control since we have always $L m_{\pi}^{v a l} \geq 4.8$. Of course this is payed with a very low UV cutoff, and one expects large lattice artifacts. These are taken into account in the analysis. 

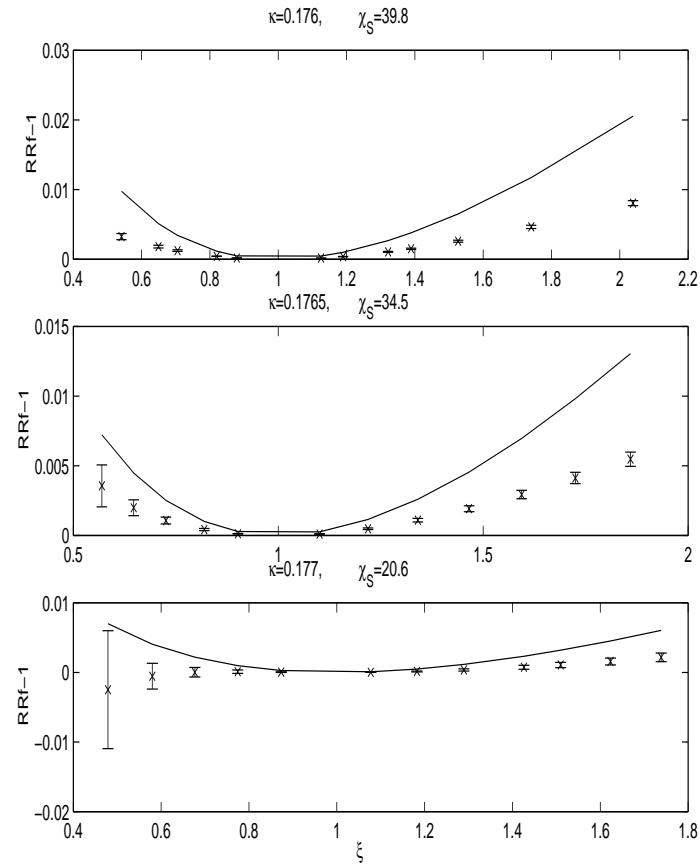

Figure 1. $R R f-1$ predictions against the data.

\section{Comparison with ChPT}

Our first goal is to confront the results of numerical simulations with the (PQ)ChPT formulas 61 . In order to cancel the $Z$-factors of multiplicative renormalization, which in the case of a mass-independent renormalization scheme only depend on the gauge coupling and not on the quark mass, we considered ratios of quark masses $\left(m_{q A}\right)$, pion masses $\left(m_{A B}\right)$ and pion decay constants $\left(f_{A B}\right)$. Here $A, B$ stand for the flavor indices of valence $(V)$ or sea $(S)$ quarks.

If we assume that there are no lattice artifacts, no NNLO corrections and we take for $\chi_{S}=$ $\frac{2 B_{0} m_{q S}}{f_{0}^{2}}$ its tree level estimate, $\chi_{S}^{e s t} \equiv \frac{\left(r_{0} m_{\pi}\right)^{2}}{\left(r_{0} f_{0}\right)^{2}}$, then the ratios (here $\xi \equiv \frac{\chi_{V}}{\chi_{S}}$ )

$R R f \equiv \frac{f_{V S}^{2}}{f_{V V} f_{S S}}=1+\frac{\chi_{S}^{e s t}}{32 N_{s} \pi^{2}}[\xi-1-\log (\xi)]$,
$R R n \equiv \frac{4 \xi m_{V S}^{4}}{(\xi+1)^{2} m_{V V}^{2} m_{S S}^{2}}=1+\frac{\chi_{S}^{e s t}[\log (\xi)-\xi+1]}{16 N_{s} \pi^{2}}$

are non trivial and parameterless predictions of ChPT. This provides a strong check of how far we
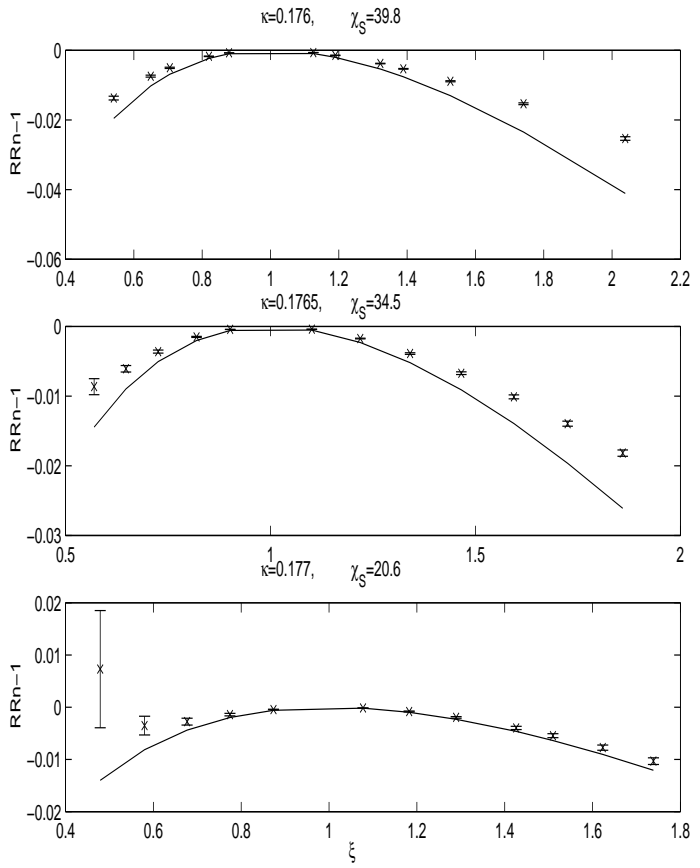

Figure 2. $R R n-1$ predictions against the data.

are from the NLO ChPT regime. In figure 1 and 2 we superimpose the predicted functions to the data. The agreement is of course not complete (there are indeed $O(a)$ and NNLO effects), but the corrections are sub-dominant contributions. Moreover the agreement improves when the sea quark masses decrease.

Encouraged by this results, we systematically compared our data with those ratios of pion masses and coupling constants which determine the Gasser-Leutwyler 7 coefficients $L_{4}, L_{5}, L_{6}$ and $L_{8}$. Since we expected large lattice artifacts in the data, the comparison was done with $\mathrm{W}(\mathrm{PQ}) \mathrm{ChPT}$ [8], including $O(a)$ lattice artifacts in the effective continuum theory. Besides that, we also included the relevant contributions from NNLO ChPT [9]. Although this involve many parameters, one can obtain enough constraints from Partially Quenched simulations. The details of the fitting procedure and the results are described in 3. Here we simply add that, following the analysis of 10, we can now include also $O\left(a^{2}\right)$ lattice artifacts in our fit of the pion mass ratios. 


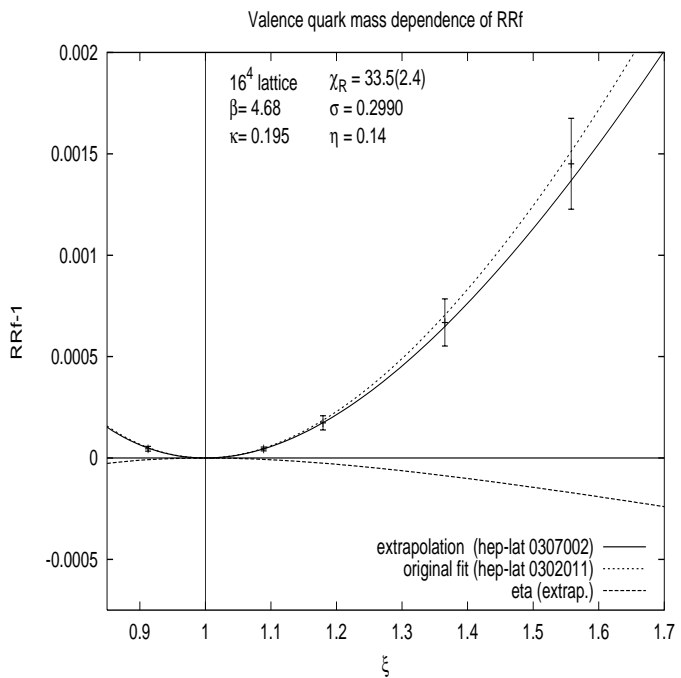

Figure 3. Comparison of the extrapolation of the fit of $R R f$ from $\beta=5.1[3$ ] with the original fit.

It turns out that this does not add new parameters to the fit, but it amounts to a redefinition of the Wilson-ChPT coefficients $W_{i}$.

In order to have a consistency check of the surprisingly small lattice artifacts that we found, we compared the results of 3 with those obtained in an older simulation with larger lattice spacing $(\beta=4.68, a \simeq 0.27 \mathrm{fm})$ 11]. The comparison (fig. [3and (4) shows quite small scale breaking. Larger scale breaking $(\sim 8 \%)$ are observed for the ratio $m_{\pi} / m_{\rho}$, which at fixed $M_{r}=1.088$ goes from 0.613 (at $\beta=5.1$ ) to 0.664 (at $\beta=4.68$ ).

To summarize: we showed that it is possible to simulate, with reasonable costs, very light $\mathrm{dy}$ namical quarks. Compensating $O(a)$ effects by introducing $O(a)$ terms in the PQCh-Lagrangian is, in this case, a viable alternative to $O(a)$ improvement of the action. The observed $O(a)$ contributions in the light Goldstone boson sector are surprisingly small, while NNLO are still important. The expected behavior predicted by PQChPT is already visible, although a quantitative determination of the LEC's still needs further simulations at smaller masses and lattice spacing. Most of the numerical calculations presented here have been done at the computers of NIC-Juelich and Zeuthen.

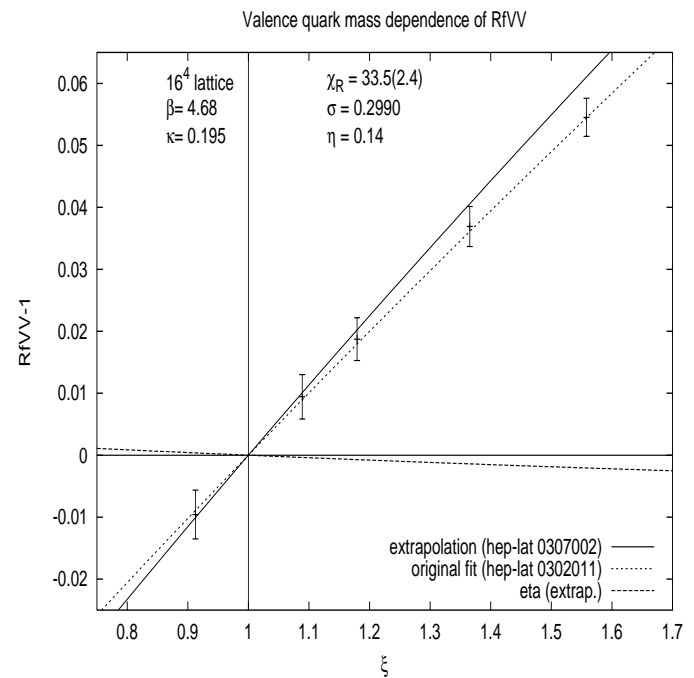

Figure 4. Comparison of the extrapolation of the fit of $R f_{V V}$ from $\beta=5.1[3]$ with the original fit.

\section{REFERENCES}

1. S.R. Sharpe, N. Shoresh, Phys. Rev. D62 (2000) 094503; hep-lat/0006017

2. S. Dürr, hep-lat/0208051.

3. qq+q Collaboration, F. Farchioni, I. Montvay, E. Scholz and L. Scorzato, To appear in Eur. Phys. J.; hep-lat/0307002.

4. I. Montvay, Nucl. Phys. B466 (1996) 259; hep-lat/9510042

5. qq+q Collaboration, F. Farchioni, C. Gebert, I. Montvay and L. Scorzato, Eur. Phys. J. C26 (2002) 237; hep-lat/0206008

6. C.W. Bernard, M.F.L. Golterman, Phys. Rev. D49 (1994) 486; hep-lat/9306005

7. J. Gasser and H. Leutwyler, Annals Phys. 158 (1984) 142.

8. G. Rupak, N. Shoresh, Phys. Rev. D66 (2002) 054503; hep-lat/0201019

9. S.R. Sharpe, R. Van de Water, these proceedings; hep-lat/0308010

10. O. Baer, G. Rupak and N. Shoresh, hep-lat/0306021.

11. qq+q Collaboration, F. Farchioni, C. Gebert, I. Montvay, E. Scholz and L. Scorzato, Phys. Lett. B561 (2003) 102; hep-lat/0302011 